\title{
Heart Rate and Blood Pressure Estimation from Compressively Sensed Photoplethysmograph
}

\author{
Pawan K. Baheti \\ Qualcomm Inc. \\ 5775 Morehouse Drive \\ San Diego, CA 92121 \\ pbaheti@qualcomm.com
}

\author{
Harinath Garudadri \\ Qualcomm Inc. \\ 5775 Morehouse Drive \\ San Diego, CA 92121 \\ hgarudad@qualcomm.com
}

\begin{abstract}
In this paper we consider the problem of low power $\mathrm{S}_{p} \mathrm{O}_{2}$ sensors for acquiring Photoplethysmograph (PPG) signals. Most of the power in $\mathrm{S}_{p} \mathrm{O}_{2}$ sensors goes to lighting red and infra-red LEDs. We use compressive sensing to lower the amount of time LEDs are lit, thereby reducing the signal acquisition power. We observe power savings by a factor that is comparable to the sampling rate. At the receiver, we reconstruct the signal with sufficient integrity for a given task. Here we consider the tasks of heart rate (HR) and blood pressure (BP) estimation. For BP estimation we use ECG signals along with the reconstructed PPG waveform. We show that the reconstruction quality can be improved at the cost of increasing compressed sensing bandwidth and receiver complexity for a given task. We present $\mathrm{HR}$ and BP estimation results using the MIMIC database.
\end{abstract}

\section{Keywords}

BAN, Pulse oximeter, Low power sensors, Compressed sensing, Matching pursuit, Blood pressure

\section{INTRODUCTION}

Body area networks (BAN) are promising for healthcare applications such as continuous monitoring for diagnostic purposes, effects of medicines on chronic ailments, etc. in addition to promoting life styles aimed at prevention and fitness. BAN consists of wireless sensors that sense the vital signs and communicate them to an aggregator such as a cell phone or PDA [1, 2]. Figure 1 illustrates a simple representation of an example BAN. The sensors used in this example are electrocardiogram (ECG) electrodes, pulse oximeters, 3D-accelerometer and microphone and the resulting sensory data could provide vital signs like heart rate, $\mathrm{S}_{p} \mathrm{O}_{2}$ (oxygen content in blood), posture/activity and respiration rate. It is desirable for such sensors to be nonintrusive and long lasting. In this work we are interested in reducing the power consumption for the pulse oximeter

Permission to make digital or hard copies of all or part of this work for personal or classroom use is granted without fee provided that copies are not made or distributed for profit or commercial advantage and that copies bear this notice and the full citation on the first page. To copy otherwise, to republish, to post on servers or to redistribute to lists, requires prior specific permission and/or a fee.

BodyNets 2009 Los Angeles, California, USA

Copyright 2008 ICST 978-963-9799-41-7 ...\$5.00.

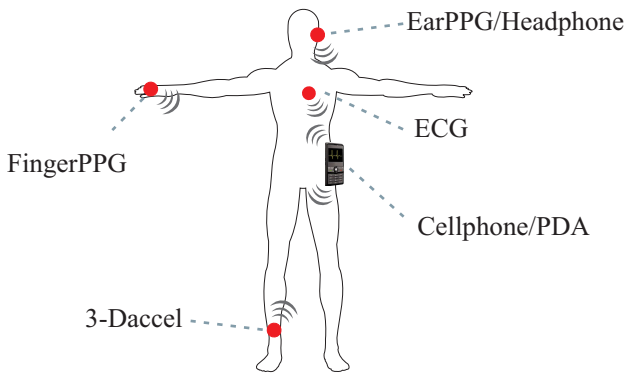

Figure 1: Example Body Area Network.

sensors. A pulse oximeter sensor generates Photoplethysmogram (PPG) waveforms, used to estimate blood oxygenation $\left(\mathrm{S}_{p} \mathrm{O}_{2}\right)$ and heart rate $(\mathrm{HR})$. In addition, $\mathrm{PPG}$ and ECG signals can be combined to estimate systolic and diastolic blood pressures (SBP and DBP) [3]. Commercial pulse oximeters consume power in the order of $20-60 \mathrm{~mW}$. The red and infrared LEDs account for most of this power [4]. Rhee et al. report a power-efficient design for a "finger-ring" PPG sensor, bringing the power consumption down to $1.5 \mathrm{~mW}$. They reduce the duty cycle for LED lighting for a given uniform sampling rate. They report use of fast detectors and higher clock frequencies among other optimizations. Thus, the LED will be switched on for $T \cdot f_{s}$ duration, where $f_{s}$ and $T$ represent the sampling rate and duration of lighting (to acquire each sample) respectively.

In this work we make use of compressed sensing (CS) [5, 6 ] framework to further reduce the power consumption of pulse oximeter sensors. We assume that the PPG signals are sparse in some transform space and hence compressible. We sample PPG at non-uniform time intervals, but with an average sampling rate of $F_{s}$. In the compressed sensing approach, $F_{s}$ is much smaller than the uniform sampling rate $f_{s}$. We refer to the factor $f_{s} / F_{s}$ as the under-sampling ratio (USR). Note that this approach will result in reduced power consumption (approximately by a factor of USR) by pulse oximeter sensors for PPG acquisition. This is because the LED is now lit up only $f_{s} /$ USR times for PPG acquisition. A benefit of this CS based approach compared to low-pass filtering and sampling at $f_{s} /$ USR is that the signal content above $f_{s} /$ USR will not be lost. Similarly narrow-band signals at higher frequencies can also be acquired with high USR. Figure 2 shows an example spectrum of a PPG signal sampled at $f_{s}=125 \mathrm{~Hz}$. It can be seen that significant spectral content will be lost if we just low-pass filter the 
PPG signal and sample at $f_{s} / 40=3.125 \mathrm{~Hz}$ for a USR of 40. Another benefit from using the CS framework is that the measurements are independent of the transform space used at reconstruction, including the Fourier space as with traditional uniform sampling. This CS measurement framework translates into significant power-savings at the sensor side, at the cost of an increase in computational overhead at the receiver to reconstruct an approximation of the PPG signal. In the context of BAN, this is desirable as we shift computational complexity to nodes with flexible power budgets and increase life of the sensor. Here we are interested in understanding the receiver complexity for a given task. For example, heart rate (HR) estimation task may not require an intermediate representation of the PPG signal from CS samples, and thus the post-processing complexity will be different for PPG signal reconstruction versus HR estimation. We also report some results in this paper demonstrating that the BP estimation accuracy is not compromised with high values of USR.

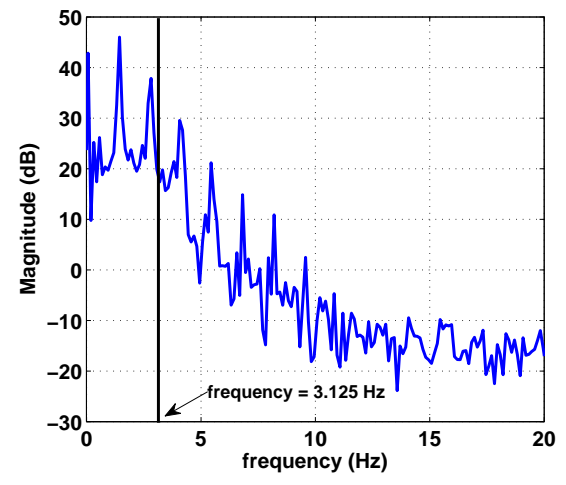

Figure 2: Example spectrum of PPG signal.

The remainder of the paper is organized as follows. In Section 2, we present an overview of uniformly sampled PPG (Nyquist-PPG) waveforms from pulse oximeter sensors and follow up with the CS principles for acquiring and reconstructing PPG (CS-PPG). Section 3 compares the complexity of the CS based post-processing (at the aggregator) for the tasks of HR estimation and complete PPG signal reconstruction. In Section 4, we discuss briefly the BP estimation algorithm based on ECG and PPG signals. We then present $\mathrm{BP}$ estimation results using Nyquist-PPG and CS-PPG for selected subjects from the MIMIC database. We present conclusions in Section 5.

\section{CS BASED ACQUISITION}

In this section we will begin by providing a brief background on conventional PPG signal acquisition, and then extend it to the CS framework. There are three main components of the PPG sensor: 1) Red and Infrared LED, 2) Photodetector and 3) Lighting and sampling sequence for LED and photodetector respectively. Figure 3 illustrates the system flow diagram for PPG sensing. The light from the LED is transmitted/reflected from the tissue and is collected on the photodetector. The ratio of the the average intensities corresponding to the red and the infrared LEDs, measured at the photodetector, is useful in determining the oxygen content $\left(\mathrm{S}_{p} \mathrm{O}_{2}\right)$ in blood. Thus, $\mathrm{S}_{p} \mathrm{O}_{2}$ is a function of the mean (DC content) of the PPG signal. The modulations in the PPG waveform (associated with either LED) are related to the instantaneous blood flow [7]. Instantaneous heart rate (HR) can be estimated as the inverse of the distance between peaks and is expressed in the units of beats per minute (BPM). The lighting sequence for the LED depends upon the desired sampling rate for the PPG signal. Up to now, we observe an uniform, Nyquist sampling rate, and note that frequent lighting of the LEDs results in significant power consumption by the pulse oximeter sensor.

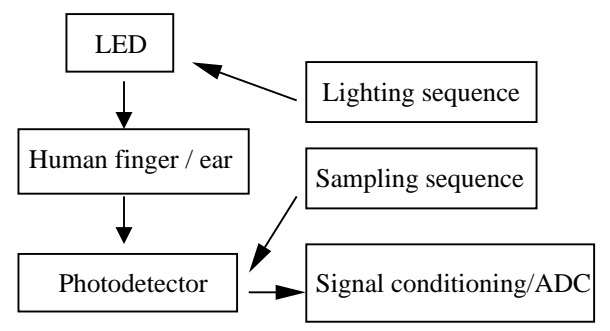

Figure 3: System flow diagram for PPG acquisition.

In this work, we are interested in exploiting the sparse nature of the PPG signal and make fewer measurements in order to save sensor power. Currently we make use of Gabor basis, consisting of various cosine waves with time support limited by Gaussian window functions at different scales, as the transform space. Let the original sampled PPG signal be denoted by $N$-dimensional vector $\mathbf{x}$ and the sparse-domain transform basis be represented by $N \times N$ matrix W. The $(i, j)$ entry of matrix $\mathbf{W}$ is given by,

$$
\begin{aligned}
{[\mathbf{W}]_{i, j}=} & \cos \left(\frac{2 \pi(i-1)(j-1)}{2 N}\right) \\
& \times \exp \left(-\frac{(i-1)^{2}(j-N / 2)^{2}}{w N^{2}}\right) .
\end{aligned}
$$

The term $w$ is associated with the width of the Gaussian kernel in the Gabor basis. We normalize each row of the matrix W such that the corresponding $L_{2}$-norm is equal to 1 , and we will refer to $\mathbf{W}$ as the sparse-basis. The PPG signal $\mathbf{x}$ is projected on the sparse-basis to generate the corresponding $N$-dimensional representation in transform space and it is given by

$$
\mathbf{y}=\mathbf{W} \mathbf{x} .
$$

Figs. 4(a) and 4(b) illustrate an example with a short segment of PPG signal and the corresponding representation $\mathbf{y}$ in transform space respectively. The figure shows a 8 second segment sampled at $125 \mathrm{~Hz}$ (i.e., $N=1000$ ). We observe that the signal $\mathbf{x}$ is sparse/compressible in the transform domain with about 30 coefficients greater than 0.2 in terms of absolute magnitude. This indicates that most of the PPG signal characteristics reside in a much lowerdimensional space compared to $N$ and thus it is compressible. This motivates us to use the recently developed CS principles by Candes and Donoho [5, 6] which allows us to make $K \ll N$ measurements (i.e. heavily under-sample the true data) and still be able to estimate $\mathbf{x}$ with high fidelity. The result by Candes et al. states that if $\mathbf{x}$ is explicitlysparse with only $M$ non-zero elements in some transform space then selecting $K \geq M \log N / M$ samples at random from $\mathbf{x}$ provides sufficient information, with high probabil- 
ity, to enable signal reconstruction with zero error [6]. In real situations the signal is never truly sparse and has some information content throughout the transform-space; however the number of significant components with magnitude greater than $\epsilon$, where $\epsilon<\max (\mathbf{x})$, is much smaller than $N$. In Fig. 4, $\epsilon$ was 0.2. The extensions of Candes's result to the case where $\mathbf{x}$ is not explicitly-sparse has been presented in [6]. The CS paradigm still remains valid; however, the reconstruction error does not go to zero.

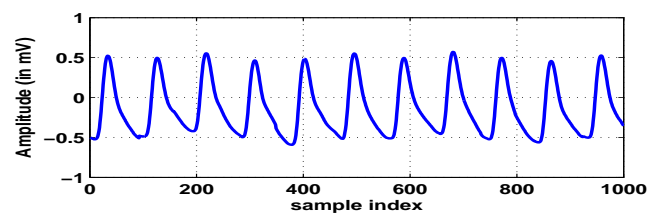

(a)

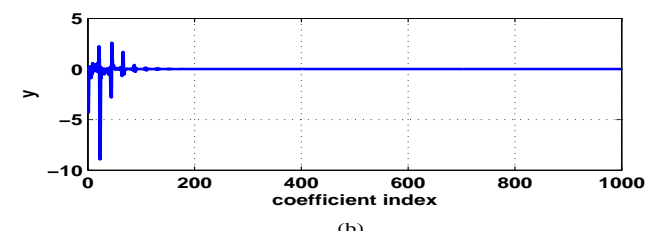

(b)

Figure 4: (a) Sample PPG signal with $N=1000$, (b) Transform of the signal from (a) in Gabor space.

We now express mathematically the sensing process for $\mathbf{x}$. Let $\mathbf{P}$ denote a $K$-dimensional vector containing unique entries (chosen at random) with each element bounded between 1 and $N$. This essentially provides the $K$ random locations to select the elements from $\mathbf{x}$. We can now write the $K$-dimensional measurement vector $\mathbf{r}$, obtained from $\mathbf{x}$, as

$$
\mathbf{r}=\mathbf{H x}
$$

where $\mathbf{H}$ denotes the $K \times N$ measurement matrix. The $i^{t h}$ row of the matrix $\mathbf{H}$ is an all-zero vector with 1 at the location given by the $i^{t h}$ element of $\mathbf{P}$. We make use of the matching pursuit (MP) algorithm for signal reconstruction from measurement vector $\mathbf{r}$. The MP technique is a greedy algorithm that builds up a signal approximation iteratively by making locally optimal decisions $[8,9]$. Here is the description of MP algorithm as described in [8].

The term $m$ is the upper-bound on the number of iterations that is allowed for reconstruction, and the term $\epsilon$ defines the convergence criterion. The simple intuition behind the above algorithm is twofold: At each iteration step, the algorithm 1) attempts to find the column of $\mathbf{V}$ that is most strongly correlated with the residual of $\mathbf{r}$, and 2) then subtracts the contribution of this column vector from $\mathbf{r}$. This algorithm is greedy in nature because at each step it estimates the most dominant component of the original signal $\mathbf{x}$ in the projection space $\mathbf{W}$. We note that the main complexity of the MP algorithm lies in step 2 which costs $O(K N)$ arithmetic operations for a single iteration [9].

We now present some reconstruction examples generated using the CS approach. We select an 8 second segment from the MIMIC database, sampled at $125 \mathrm{~Hz}$ (i.e., $N=1000$ ). Recall that the number of CS samples is $K$ that defines our under-sampling ratio (USR) as $N / K$. Figure 5 demonstrates the example CS-PPG signal reconstructions obtained for $\mathrm{USR}=10,20$ and 30 with the upper bound on the number Algorithm: MP algorithm for reconstruction from CS
measurements

1. Initialization: Define a modified basis $\mathbf{V}=\mathbf{H W}$ (of dimension $K \times N)$ such that $\mathbf{V}=\left[\mathbf{V}_{1} \ldots \mathbf{V}_{N}\right]$ where $\mathbf{V}_{j}$ is the $j^{\text {th }}$ column vector of $\mathbf{V}$. Initialize the residual $\mathbf{r}_{0}=\mathbf{r}$ and the approximation $\hat{\mathbf{y}}=0$. The dimension of $\hat{\mathbf{y}}$ is same as $\mathbf{y}$ (i.e., $N$ ). Initialize the iteration counter $i=1$.

2. Find the column vector from $\mathbf{V}$ that maximizes the innerproduct of the residual $\mathbf{r}_{i-1}$ onto $\mathbf{V}$

$$
n_{i}=\arg \max _{j=1 \ldots N} \frac{\left\langle\mathbf{r}_{i-1}, \mathbf{V}_{j}\right\rangle}{\left\|\mathbf{V}_{j}\right\|_{L_{2}}} .
$$

3. Update the residual and the estimate of coefficient vector $\mathbf{y}$ as follows

$$
\begin{aligned}
\mathbf{r}_{i} & =\mathbf{r}_{i-1}-\frac{\left\langle\mathbf{r}_{i-1}, \mathbf{V}_{n_{i}}\right\rangle}{\left\|\mathbf{V}_{n_{i}}\right\|_{L_{2}}^{2}} \mathbf{V}_{n_{i}} \\
\hat{\mathbf{y}}_{n_{i}} & =\hat{\mathbf{y}}_{n_{i}}+\frac{\left\langle\mathbf{r}_{i-1}, \mathbf{V}_{n_{i}}\right\rangle}{\left\|\mathbf{V}_{n_{i}}\right\|_{L_{2}}^{2}}
\end{aligned}
$$

4. Increment $i$, Continue and go to step 2 if $i<m$ and $\left\|\mathbf{r}_{i}\right\|_{L_{2}}>\epsilon\|\mathbf{r}\|_{L_{2}}$ otherwise go to next step.

5. Obtain the signal estimate $\hat{\mathbf{x}}=\mathbf{W} \hat{\mathbf{y}}$.

of MP iterations $(m)$ to be 500. The solid black curve represents the uniformly sampled original signal and the remaining different curves represent different values of USR. From this data we observe that the signal integrity is well preserved till $\mathrm{USR}=20$, and starts degrading thereafter. However, note that the signal peak locations are well-preserved even with high USR. It is important to realize that the LED power consumption (in the PPG data acquisition) will reduce significantly (by a factor of USR) because we are sensing the signal at much smaller rate.

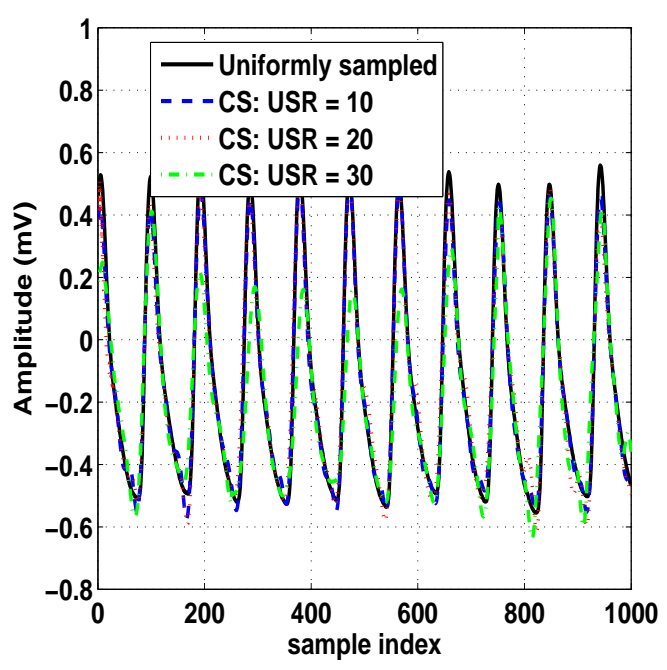

Figure 5: Comparisons of signal reconstructions obtained using USR $=10,20$ and 30 .

\section{HR AND PPG RECONSTRUCTION}


Recall from Eqns. (1) and (2) that each element of the coefficient vector $\mathbf{y}$ approximately represents the strength of a cosine (with specific frequency) in the signal $\mathbf{x}$. We also note that the PPG signal is oscillatory in nature with the dominant frequency being proportional to the HR. The $\mathrm{HR}$ can therefore be estimated from the CS reconstruction $\hat{\mathbf{y}}$ (which is obtained via MP algorithm) as

$$
\begin{aligned}
& \hat{\mathrm{HR}}=60\left(n_{\max }-1\right) \cdot \frac{f_{s}}{2 N} \mathrm{BPM}, \\
& \text { where } n_{\max }=\arg \max _{j=2 \ldots N}[\hat{\mathbf{y}}]_{j},
\end{aligned}
$$

and $[\hat{\mathbf{y}}]_{j}$ is the $j^{\text {th }}$ element of vector $\hat{\mathbf{y}}$. Note that this HR estimate is an average obtained over the time duration of $N \cdot f_{S}$ seconds. Also, the resolution of the estimate is given by $\frac{60 f_{s}}{2 N} \mathrm{BPM}$ which is equal to $3.75 \mathrm{BPM}$ for $N=1000$ and $f_{s}=125 \mathrm{~Hz}$. Consider the example PPG signal segment in Fig. 4 , where the $n_{\max }$ is found to be 23 which corresponds to $82.5 \mathrm{BPM}$. Also, recall that the MP algorithm is greedy in nature which implies that the dominant components of the PPG signal in projection space $\mathbf{W}$ are estimated in the initial iterations. In this case the two most dominant components are the DC component and the cosine with frequency corresponding to HR. Therefore, we expect to achieve a reliable estimate of HR within a few iterations of the MP algorithm, which also suggests that there is no need of generating an intermediate representation of the complete PPG signal. On the other hand we need to reconstruct more coefficients in the projection space $\mathbf{W}$ for signal reconstruction task, which results in significantly more iterations of MP postprocessing. Note that $m$ iterations in the MP algorithm cost roughly $O(m K N)$ arithmetic operations. Therefore the HR estimation task (from CS measurements) shall require less computational complexity at the receiver when compared to the signal reconstruction task. Also recall that the number of CS samples corresponding to a $N$-dimensional signal $\mathbf{x}$ is $K$, which defines our under-sampling ratio (USR) as $N / K$. With increasing $U S R$ we would expect the computational complexity to increase at the receiver in order to achieve a specified performance level. This is the trade-off between sensor power (fewer measurements at $\mathrm{Tx}$ ) and aggregator power (more iterations and computation at $\mathrm{Rx}$ ).

We now introduce the performance metrics for HR estimation and PPG signal reconstruction. For heart-rate estimation the metric is root-mean-square-error (RMSE) and it is defined as $\sqrt{E\left[\|\mathrm{HR}-\hat{\mathrm{HR}}\|^{2}\right]}$. The metric for PPG reconstruction task is normalized RMSE and it is defined as $\frac{\sqrt{E\left[\|\mathbf{x}-\hat{\mathbf{x}}\|^{2}\right]}}{\max \{|\mathbf{x}|\}}$. The term $E[\cdot]$ denotes the expectation operator with Monte Carlo averaging over various realizations of PPG signals (x) and different measurement bases $(\mathbf{H})$. The PPG signal realizations $(\mathbf{x})$ are taken from the MIMIC database. The true heart rate $\mathrm{HR}$ can be extracted from the original signal $\mathbf{x}$ as suggested in Eq. (7). Each signal segment $\mathbf{x}$ is taken to be 8 seconds long and is sampled at $125 \mathrm{~Hz}$.

Fig. 6 plots HR estimation RMSE with respect to number of iterations $m$ (for MP post-processing) for USR $=10$ and 20. We observe that RMSE decreases with increasing $m$ as the estimation accuracy improves with increasing number of iterations. Also, the number of iterations required to achieve a specified RMSE increases with increasing USR. Note that at $m=50$, the HR estimation RMSE equals 1

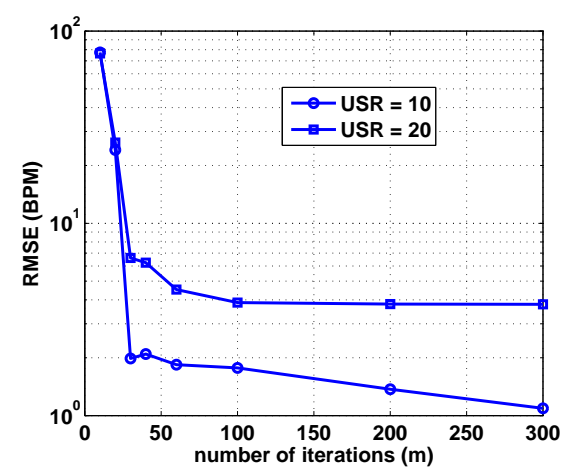

Figure 6: Comparison of HR estimation based on CS framework (for USR $=10$ and 20.)

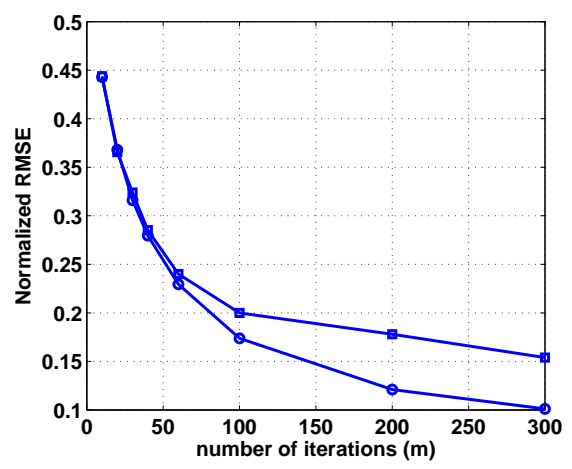

Figure 7: Comparison of PPG signal reconstruction based on CS framework (for USR $=10$ and 20.)

$\mathrm{BPM}$ and $4 \mathrm{BPM}$ for $\mathrm{USR}=10$ and 20 respectively. Therefore, in order to achieve a HR estimation RMSE of 1 BPM at $\mathrm{USR}=10$, we require roughly on the order of 0.625 million arithmetic operations per second (i.e., $O(m K N) \cdot \frac{f_{s}}{N}$, where $\left.f_{s}=125 \mathrm{~Hz}\right)$. Similarly, for reconstructing the signal with an $\mathrm{RMSE} \approx 0.1$ at $\mathrm{USR}=10$, we require roughly on the order of 3.875 million arithmetic operations per second (i.e., $\left.O\left(m K N+N^{2}\right) \cdot \frac{f_{s}}{N}\right)$. This suggests that the estimation approach described here can be easily handled by current smartphones or PDAs from the complexity standpoint. Fig. 7 plots the normalized reconstruction RMSE with respect to $m$ for $\mathrm{USR}=10$ and 20 . We first observe that the RMSE reduces with increasing $m$ as expected. However, we note that the decrease is gradual with $m$ as opposed to HR estimation RMSE. This is simply because the reconstruction task requires reconstructing more coefficients in the sparse space $\mathbf{W}$ as opposed to a single dominant component in the case of HR estimation. To summarize, the receiver complexity depends upon USR, the task of interest and desired performance level. An intermediate representation with full reconstruction may not be needed for some tasks such as HR estimation from PPG.

\section{CUFFLESS BP ESTIMATION}

In this section we consider the application of cuffless blood pressure estimation based on the PPG signal reconstructed using CS framework (i.e., CS-PPG) and ECG signal. It has 


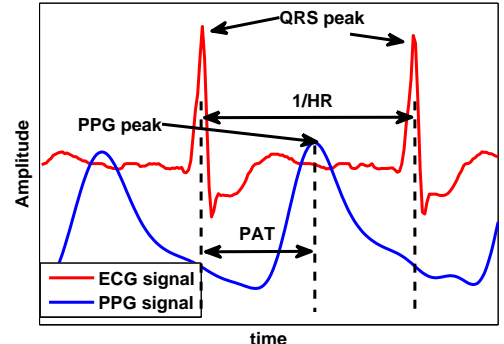

Figure 8: Measurement of PAT and HR through ECG and PPG signal peaks.

been previously shown in [3] that $\mathrm{SBP}$ and $\mathrm{DBP}$ can be estimated using Pulse Arrival Time (PAT), where PAT is defined as the delay between QRS peak in ECG waveform and corresponding peak in PPG waveform. Here we use a baseline BP estimation algorithm that uses PPG, ECG and heart rate (HR), to investigate the applicability of CS-PPG for BP estimation. Fig. 8 illustrates the definition of PAT and HR through example ECG and PPG waveforms. Our baseline BP estimation model in can be stated as follows:

$$
\begin{aligned}
\mathrm{SBP} & =a_{1} \cdot \mathrm{PAT}+b_{1} \cdot \mathrm{HR}+c_{1}, \\
\mathrm{DBP} & =a_{2} \cdot \mathrm{PAT}+b_{2} \cdot \mathrm{HR}+c_{2},
\end{aligned}
$$

where the calibration parameters $a_{i}, b_{i}$ and $c_{i}, i=1,2$ are estimated during training process and adapted through recursive least squares (RLS) algorithm at every re-calibration opportunity. In this work, re-calibration is done once every 1 hour. Re-calibration duration essentially implies how often one needs the ground truth to estimate/adapt the model parameters. The re-calibration step is required for a real application to tackle with bias and drift issues. We would like the re-calibration period to be longer so that blood pressure can be measured continuously and non-invasively in a cuffless manner for longer periods. More frequent re-calibrations reduce the $\mathrm{BP}$ estimation error whereas less frequent recalibrations will make the system more amenable for everyday use. Reducing re-calibration frequency, while maintaining BP estimation accuracy is an active area of research. By identifying parameters that account for drift/bias and incorporating them in the $\mathrm{BP}$ estimation model, it may be possible to reduce re-calibration further.

In order to have reliable PAT and HR estimates at any instant, one has to make sure that signal peaks in the vicinity are reliable. There are many regions in the MIMIC patient records where peak locations are ambiguous. Our peak-extraction algorithm discards the data segments with unreliable ECG and/or PPG signals.

Next, we present results of BP estimation using CS-PPG and compare them with those using Nyquist-PPG. The records corresponding to 13 patients from the MIMIC database are used for this evaluation. The length of the records for these patients is on average of the order of 38 hours. The gradient projection based sparse reconstruction (GPSR) approach described in [10] is used to generate CS-PPG for the entire patient record. Note that we are using a different reconstruction approach than the algorithm described in previous section. We used MP algorithm in the previous section because it is more amenable from the standpoint of complexity analysis. Table 1 presents BP estimation results based on the use of ECG and CS-PPG signals of our approach. The value of USR is taken to be 40 for the CS framework based measurement of the PPG signal. It is important from BP monitoring perspective to compute the frequency of reporting/generating BP estimates. To do so we introduce the term $N_{B P}$ as average number of BP estimates generated per minute using CS-PPG and the Nyquist-PPG baseline algorithm. Table 1 shows the standard deviation (s.d.) of the SBP and DBP estimation error and $N_{B P}$ for NyquistPPG and CS-PPG (with USR=40). The Association for the Advancement of Medical Instrumentation (AAMI) requirements for $\mathrm{BP}$ estimation indicate that the standard deviation of the error has to be below $8 \mathrm{mmHg}$, both for SBP and DBP. Note that the average s.d. for both SBP and DBP estimation error is less than $8 \mathrm{mmHg}$. Furthermore the accuracy does not degrade by using CS-PPG when compared to Nyquist-PPG. In this simulation study we estimate an average of $8.85 \mathrm{BP}$ measurements per minute at $\mathrm{USR}=40$, compared with an average of 51 BP estimates per minute using Nyquist-PPG.

\begin{tabular}{|c|c|c|c|c|c|c|}
\hline & \multicolumn{3}{|c|}{ Nyquist-PPG } & \multicolumn{2}{c|}{ CS-PPG (USR=40) } \\
\hline Patient & $\begin{array}{c}\text { SBP } \\
(\mathbf{m m H g})\end{array}$ & $\begin{array}{c}\text { DBP } \\
(\mathbf{m m H})\end{array}$ & $N_{B P}$ & $\begin{array}{c}\text { SBP } \\
\mathbf{m m m} \text { m }\end{array}$ & $\begin{array}{c}\text { DBP } \\
(\mathbf{m m H g})\end{array}$ & $N_{B P}$ \\
\hline 55 & 4.72 & 3.1 & 48.8 & 2.95 & 2.19 & 13.05 \\
212 & 4.02 & 3.12 & 75.6 & 3.97 & 1.93 & 13 \\
213 & 4.54 & 2.27 & 21.44 & 5.54 & 2.18 & 3.3 \\
219 & 7.68 & 3.65 & 29.8 & 8.85 & 3.14 & 6.88 \\
221 & 8.24 & 3.85 & 81.5 & 6.67 & 4.03 & 10.4 \\
224 & 6.47 & 4.87 & 63.05 & 6.1 & 3.84 & 12.35 \\
230 & 6.42 & 3.3 & 37.8 & 6.09 & 3.68 & 1.52 \\
237 & 9.25 & 6.83 & 18.61 & 7.89 & 3.62 & 5.52 \\
408 & 8.99 & 9.06 & 73.41 & 8.46 & 6.66 & 12.4 \\
443 & 11.42 & 7.75 & 86.93 & 7.45 & 3.97 & 5.73 \\
444 & 7.85 & 4.08 & 59.23 & 7.49 & 4.02 & 13.1 \\
476 & 8.92 & 5.5 & 49.96 & 7.06 & 2.49 & 15.02 \\
482 & 7.34 & 2.97 & 16.92 & 6.1 & 2.35 & 2.83 \\
Avg: & 7.37 & 4.64 & 51 & 6.51 & 3.39 & 8.85 \\
\hline
\end{tabular}

Table 1: Standard deviation (s.d.) for SBP, DBP estimation error and $N_{B P}$ for Nyquist-PPG and CS$\mathbf{P P G}$ at $\mathbf{U S R}=40$.

\section{CONCLUSIONS}

We presented a compressed sensing based low power solution to acquire PPG signals. The power consumption at the sensor is reduced by making use of CS measurement framework. We observed that the LED power consumption (in the PPG data acquisition) reduces significantly (by a factor of USR) because the signal is sensed at much smaller rate. This however results in increase of post-processing complexity at the receiver. In this work we analyzed the receiver complexity for tasks like heart rate estimation and PPG signal reconstruction. In the current version the receiver complexity is defined in terms of arithmetic operations/computations used for estimating HR and BP. The limitation of the current definition is that it only accounts for the algorithmic complexity. HR estimation does not require the intermediate representation of the PPG signal which therefore results in reduced post-processing complexity when compared to the PPG signal reconstruction task. We also make use of CS-PPG and ECG signals to perform cuffless-BP estimation. The under-sampling ratio of 40 was used for measurement purposes and CS based reconstruction. We presented SBP and DBP estimation results comparing Nyquist-PPG and CS-PPG (with USR=40). We found that the CS-PPG signal results in average SBP and DBP standard deviations of $6.51 \mathrm{mmHg}$ and $3.39 \mathrm{mmHg}$, respectively. 


\section{REFERENCES}

[1] U. Varshney. Pervasive healthcare and wireless health monitoring. Mobile Networks and Applications, 12:113-127, March 2007.

[2] H. B. Li and R. Kohno. Introduction of SG-BAN in IEEE 802.15 with related discussion. In IEEE International Conference on Ultra-Wideband, pages 134-139, September 2007.

[3] C. C. Y. Poon and Y. T. Zhang. Cuff-less and noninvasive measurements of arterial blood pressure by pulse transit time. In Proc. IEEE EMBS Conf., pages 5877-5880, Shanghai, China, September 2005.

[4] S. Rhee, B. Yang, and H. H. Asada. Artifact-resistant power-efficient design of finger-ring plethysmographic sensors. IEEE Transactions on Biomedical Engineering, 48:795-805, July 2001.

[5] D. Donoho. Compressed sensing. IEEE Transactions on Biomedical Engineering, 52:1289-1306, April 2006.

[6] E. Candes, J. Romberg, and T. Tao. Stable signal recovery from incomplete and inaccurate measurements. Communications on Pure and Applied Mathematics, 59:1207-1223, August 2006.

[7] R. S. Khandpur. Biomedical Instrumentation: Technology and Applications. Mc Graw Hill, NY, 2005.

[8] M. F. Duarte, M. A. Davenport, M. B. Wakin, and R. G. Baraniuk. Sparse signal detection from incoherent projections. In Proc. Int. Conf. on Acoustics, Speech, and Signal Proc. (ICASSP), May 2006.

[9] J. A. Tropp, M. B. Wakin, M. F. Duarte, D. Baron, and R. G. Baraniuk. Random filters for compressive sampling and reconstruction. In Proc. Int. Conf. on Acoustics, Speech, and Signal Proc. (ICASSP), May 2006.

[10] M. A. T. Figueiredo, R. D. Nowak, and S. J. Wright. Gradient projection for sparse reconstruction: Applications to compressed sensing and other inverse problems. IEEE Journal of Selected Topics in Signal Processing, 1:586-598, Dec 2007. 